\title{
Edge modes of surface plasmon nanodisk antennas visualized in 3-photon PEEM
}

\author{
R. C. Word ${ }^{1}$ and R. Könenkamp ${ }^{1}$ \\ ${ }^{1}$ Department of Physics, Portland State University, Portland, OR 97201, USA
}

Photoelectron emission microscopy (PEEM) provides the high spatial resolution of electron microscopy and, at the same time, the ability to explore the breadth and complexity of light-matter interactions. The emission of imaging photoelectrons is based on the photoelectric effect, which is a fundamental quantum mechanical phenomenon. As a result PEEM images provide information about the photoemission thresholds of a specimen. The rate of photoemission depends on the electric field at the surface. Additionally, ultrafast lasers permit PEEM to be used at visible and infrared energies. In this regime, the yield of multi-photon PEEM (nP-PEEM) is proportional to incident intensity to $n$ power, where $n$ is the number of photons per electron needed to overcome the photoemission threshold. PEEM thus provides a high resolution, probe-free method of studying surface electromagnetic fields. The origin of these fields can be photonic or plasmonic. PEEM can be used, for example, to study light propagating in a slab waveguide [1], the time resolved propagation of long-ranging surface plasmon polaritons (SPP) confined to a planar metal surface [2], or, as in the present case, localized surface plasmon field distributions of nanoscale antennas [3]. Such characterization of optical and plasmonic antennas is important in the quest to develop nanoscale optical communication devices [4, 5] where there is particular interest in the infrared and visible spectral region $[6,7]$.

Here we report the direct observation of plasmonic edge modes or whispering gallery-type modes of nanoscale omni-directional disk antennas using near infrared three-photon PEEM [8]. Our antennas are milled with a focused ion beam (FIB) from gold on copper composite films on indium tin oxide substrate (ITO) on glass. An ultrafast laser $\left(\lambda_{0}=800 \mathrm{~nm}\right)$ incident at $60^{\circ}$ to the surface normal generates surface plasmons at the edges of the disks by diffraction (Fig 1). Imaged photoelectrons transmit information on the strength and extent of the evanescent plasmon fields generated along the antenna rim and across its surfaces. High contrast between the pristine gold and milled ITO regions occurs because the excitation laser light energy of $1.55 \mathrm{eV}$, is sufficient for 3-photon emission from ITO (work function 4.2-4.5 eV) and possibly copper (4.5-4.8 eV), whereas four photons are needed for photoemission from gold $(5.0-5.1 \mathrm{eV})$.

It is important to stress that in these experiments a femtosecond pulsed laser is used to optically excite plasmon modes. Photoelectron emission occurs as the plasmonic excitation decays and interferes with the incident laser light. This detection process is different from the approach in EELS and cathodoluminescence where SPP modes are excited by electron beams [7, 9]. In our work resonances and constructive interference effects occur when the circumference of the receiver antenna is equal to an integer number of surface plasmon wavelengths $\left(\lambda_{\mathrm{SPP}}=775 \mathrm{~nm}\right)[10]$ as shown for a quadrupole mode in Fig 1. We have recently shown that the quadrupole field can be rotated in-plane to any desired position by controlling the angle of the incident light polarization. This control of the field position can be used to fine-tune the detection of particles in plasmonic sensor arrangements. Our experimental results agree well with the electric field distribution calculated by finite element method (FEM) simulations. Additional experiments are presently underway to observe higher and lower order modes, which FEM simulations suggest should also be observable. Of particular interest is the role the gap width, mill depth, and ITO layer plays in the development of the modes. 


\section{Experimental details}

The disk antennas were milled using a FEI-235 FIB from a composite gold/copper metal film, which was vacuum evaporated onto ITO coated glass coverslips (SPI Supplies). The gallium beam current of the FIB was $30 \mathrm{pA}$. In a home-built aberration-corrected PEEM the antennas were exposed to 800-nm, 900-mW, 80-fs laser light from a Ti:sapphire laser. The laser spot size was approximately $100 \mu \mathrm{m}$, was incident at $60^{\circ}$, and had polarization set by a tunable wave plate. Photoelectron images were recorded with a CCD optically coupled to a phosphor screen with an image exposure time of 60 seconds. The images below are composite averages made from images of six identically prepared antennas.

\section{References}

[1] JPS Fitzgerald, RC Word, R Könenkamp, Phys. Rev. B 89 (2014), p. 195129.

[2] C Lemke et al., Nano Lett. 13 (2013), p. 1053.

[3] L Douillard, F Charra, J. Electron. Spectrosc. Relat. Phenom. 189, Supplement (2013), p. 24.

[4] A Alù, N Engheta, Phys. Rev. Lett. 104 (2010), p. 213902.

[5] T Coenen, EJR Vesseur, A Polman, ACS Nano 6 (2012), p. 1742.

[6] J Lee, J Song, GY Sung, JH Shin, Nano Lett. 14 (2014), p. 5533.

[7] EJR Vesseur, A Polman, Nano Lett. 11 (2011), p. 5524.

[8] RC Word, JPS Fitzgerald, R Könenkamp, Appl. Phys. Lett. 105 (2014), p. 111114.

[9] F-P Schmidt et al., Nano Lett. 12 (2012), p. 5780.

[10] F-P Schmidt et al., Nat. Commun. 5 (2014), p. 3604.

[11] This research was supported by the US-DOE Basic Science Office under Contract No. DE-FG0213ER46406.
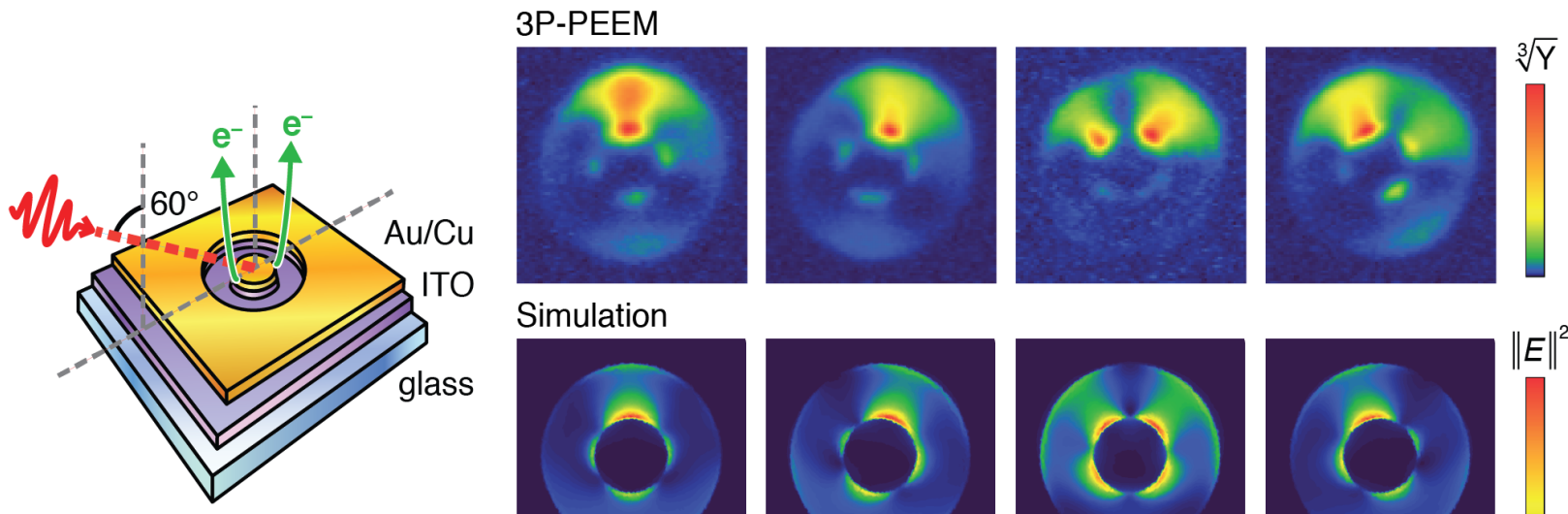

Simulation
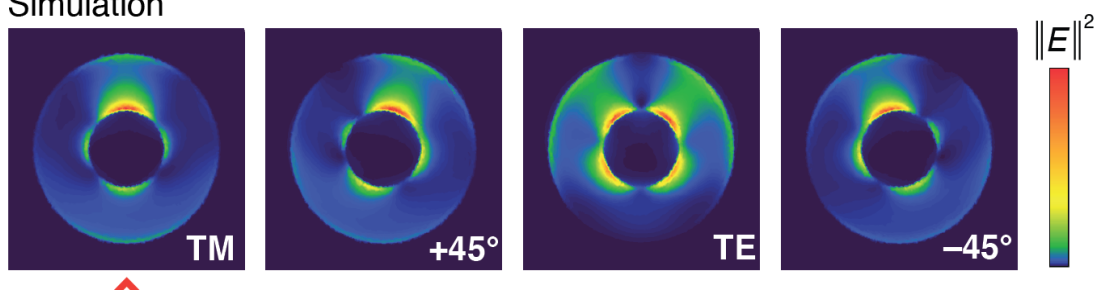

Light

$1 \mu \mathrm{m}$

Figure 1. (Left) Drawing of a gold/copper nanodisk antenna on ITO/glass substrate indicating the direction and angle of incident laser light. Photo-emitted electrons are accelerated into the PEEM's electron optics above the surface. (Right) False-color three-photon PEEM images of the quadrupole mode of an antenna (shown as cube-root of the yield) compared to FEM simulations (as electric field norm squared) for four different light polarizations. 